\title{
The Constructions of Egg-Shaped Surface Equations using Hugelschaffer's Egg-Shaped Curve
}

\author{
Ahmat Rif'an Maulana ${ }^{1 *}$, Mahmud Yunus ${ }^{2}$, and Dwi Ratna Sulistyaningrum ${ }^{3}$ \\ ${ }^{1,2,3}$ Department of Mathematics, Faculty of Mathematics and Natural Sciences \\ Sepuluh Nopember Institute of Technology \\ Kampus ITS, Keputih, Sukolilo-Surabaya 60111, Indonesia
}

Received: 8 May 2015, Revised: 6 Aughust 2015, Accepted: 29 November 2015

\begin{abstract}
Hugelschaffer's egg-shaped curve is egg-shaped curve that is constructed by two non-concentric circles using Newton's transformation known as hyperbolism. This study has goals to construct the egg-shaped surface equations using Hugelschafer's egg-shaped curve that is rotated on $x$-axis, $y$-axis and $z$-axis; to get the volume formula of the egg-shaped solid and the egg-shaped surface area and also to visualize the egg-shaped surface equations using GeoGebra. Hugelschaffer's egg-shaped curve is selected because its equation is simple. The procedures of the construction of the egg-shaped surface equations are done by drawing the curve on $x y$-plane and $x z$-plane, then it is rotated on axes of the coordinate. Whereas, the volume formula of the egg-shaped solid is gotten by using the disk method of the volume integral. The egg-shaped surface area is attained by using the integral of surface area. Visualisation of the egg-shaped surface equations are done by choosing vary of parameter values of the equations that aims to know the effect of the parameter values with the shaped surface.
\end{abstract}

Keywords: Hugelschaffer's egg-shaped curve, egg-shaped surface equations

\section{INTRODUCTION}

This paper is motivated by applications of the egg-shaped curve on egg-shaped gutters in the roof and the ground. It is explained that the cross-section of flowing water is egg-shaped, so they are said to have good drainage. In addition, the egg-shaped have been applicated on speaker design. It is explained that by suppressing various oscillations and echoes occurring inside the speaker, the eggshaped is used to pursue reproduction of the original sounds. Another application is the egg-shaped sludge digestion chambers. In comparison to previous tubular digestion chamber, it is explained that they are superior in terms of water-tightness and air-tightness [1].

Hugelschaffer's egg-shaped curve is a closed curve that represents the margin line of an egg [2] and often called Hugelschaffer's egg-shaped curve construction [3]. The idea of the construction of Hugelschaffer's egg-shaped curve is constructive

\footnotetext{
Corresponding author.

E-mail address: ahmatrifan@gmail.com
}

procedure of Newton's transformation that is done by two non-concentric circles, known as hyperbolism [4]. The Hugelschaffer's egg-shaped curve has mathematically simple equation, that is identically to an equation of distortion ellips, but the equation is bounded on two dimension [5]. The equation of Hugelschaffer's egg-shaped curve has three shaped parameters. They play important role on the shaped of the egg-shaped. These parameters are $a, b$ and $w$. If we change the values of the parameter, we can obtain oval egg-shaped, pyriform egg-shaped, circular and elliptical egg-shaped [6].

According to these reasons, in this paper, the equation of Hugelschaffer's egg-shaped curve will be used to construct the egg-shaped surface equation (3D egg-shaped equation) and analyse the effects of the parameter values of the egg-shaped surface equation.

\section{THE EQUATION OF HUGELSCHAFFER'S EGG-SHAPED CURVE}

The construction of Hugelschaffer's eggshaped curve can be obtained from two non- 
concentric circles using definition of Newton's transformation [7]. That is, we make sketch of circle $k_{1}$ with radius $a$ and circle $k_{2}$ with radius $b$, where the center point of circle $k_{1}$ and circle $k_{2}$ have distance $w$. It is shown in Figure 1.

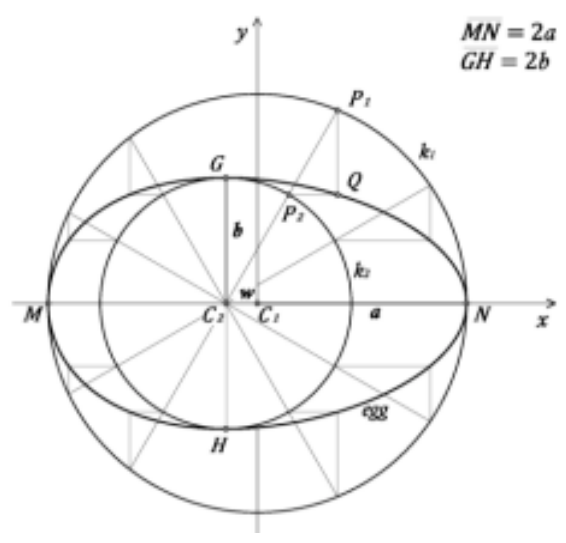

Fig. 1. Construction of Hugelschaffer's egg-shaped curve.

Furthermore, by using the comparison of gradient $C_{2} P_{2}$ and $C_{2} P_{1}$, we obtained the equation of Hügelchaffer's egg-shaped curve, that is

$\frac{x^{2}}{a^{2}}+\frac{y^{2}}{b^{2}}\left(1+\frac{2 w x+w^{2}}{a^{2}}\right)=1$,

with $a>b>0$ and $0<|w|<a ; a, b, w$ constants and $M N$ is major axis of curve and $G H$ is minor axis of curve [7].

\section{RESEARCH METHOD}

The finding processes of the egg-shaped surface equations are done by making sketch of the egg-shaped curve on $x y$-plane and $x z$-plane. Then, the curve is rotated on the axes of coordinate. Whereas, the volume formula of egg-shaped solid is attained by using disk method of the volume integral [8]. The egg-shaped surface area is found using integral of surface area that is numerically solved [9]. Visualisation of the egg-shaped surface equation is done by using GeoGebra where the equation must be changed to parametric form.

\section{RESULTS AND DISCUSSION}

The construction of the egg-shaped surface equations with $x$-axis as rotation axis

The construction steps are as follow. First, we make sketch of Hügelschaffer's egg-shaped curve ( $T$ curve) in $x y$-plane where the major axis of the curve is on $x$-axis and the mid-point of the major axis is at the point $O(0,0,0)$. It is shown in the Figure 2 .

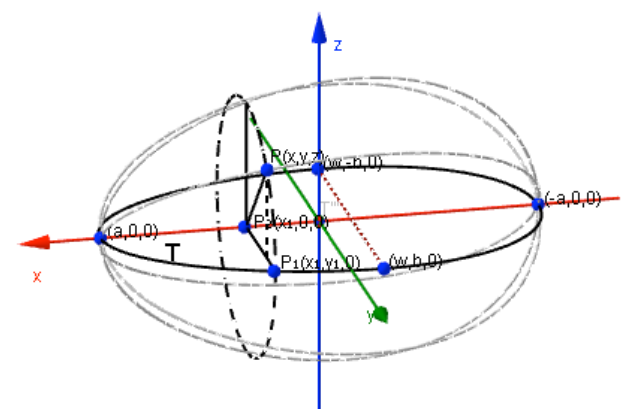

Fig. 2. The $T$ curve on $x y$-plane rotated on $x$-axis.

Second, we take any point on $T$ curve, let $P_{1}\left(x_{1}, y_{1}, 0\right)$, and we state the equation of $T$ curve at the point $P_{1}$. Third, the $T$ curve is rotated on $x$-axis, so that the point $P_{1}\left(x_{1}, y_{1}, 0\right)$ forms circle path with point $P_{2}\left(x_{1}, 0,0\right)$ as centre point and radius $\left|P_{2} P_{1}\right|=y_{1}$. Fourth, we take any point on circle path, let $P(x, y, z)$. Then, we show that the point $P(x, y, z)$ is satisfy the equation of $T$ curve in the point $P_{1}$, that is by using the relations of radius $\left|P_{2} P\right|$ and $\left|P_{2} P_{1}\right|$, we project the point $P(x, y, z)$ on $x y$-plane, so that we get the egg-shaped surface equation where the mid-point of major axis is at $O(0,0,0)$, that is

$\frac{x^{2}}{a^{2}}+\frac{y^{2}}{b^{2}}\left(1+\frac{2 w x+w^{2}}{a^{2}}\right)+\frac{z^{2}}{b^{2}}\left(1+\frac{2 w x+w^{2}}{a^{2}}\right)=1$,

where $a>b>0 ; 0<|w|<a ; a, b, w$ constants.

Whereas, the egg-shaped surface equation where the mid-point of the major axis is at $Q(p, q, r)$, that is

$\frac{(x-p)^{2}}{a^{2}}+\frac{(y-q)^{2}}{b^{2}} f(x)+\frac{(z-r)^{2}}{b^{2}} f(x)=1$

where $f(x)=\left(1+\frac{2 w(x-p)+w^{2}}{a^{2}}\right)$.

The construction of the egg-shaped surface equations with $y$-axis as rotation axis

The construction steps are similar to the construction of the equation of egg-shaped surface where the $x$-axis as rotation axis. The difference step is only on the first step, that is, we make sketch of $T$ curve on $x y$-plane where the major axis curve is on the $y$-axis. It is shown in the Figure 3 . 


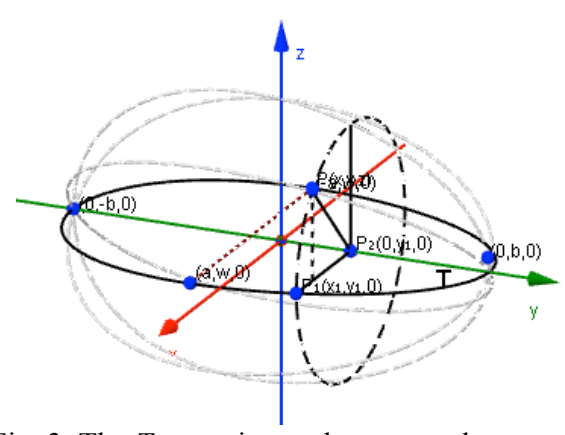

Fig. 3. The $T$ curve in $x y$-plane rotated on $y$-axis.

Then, we obtained the equation of egg-shaped surface, where the mid-point of major axis is at $O(0,0,0)$ and the $y$-axis is as rotation axis, that is,

$$
\frac{x^{2}}{a^{2}}\left(1+\frac{2 w y+w^{2}}{b^{2}}\right)+\frac{y^{2}}{b^{2}}+\frac{z^{2}}{a^{2}}\left(1+\frac{2 w y+w^{2}}{b^{2}}\right)=1,
$$

where $b>a>0 ;<0|w|<b ; a, b, w$ constants.

Whereas, the equation of egg-shaped surface where the mid-point of major axis at $Q(p, q, r)$ is

$\frac{(x-p)^{2}}{a^{2}} f(y)+\frac{(y-q)^{2}}{b^{2}}+\frac{(z-r)^{2}}{a^{2}} f(y)=1$

with $f(y)=\left(1+\frac{2 w(y-q)+w^{2}}{b^{2}}\right)$.

The construction of egg-shaped surface equations with z-axis as rotation axis

If we make sketch $T$ curve on $x z$-plane that is shown in the Figure 4.

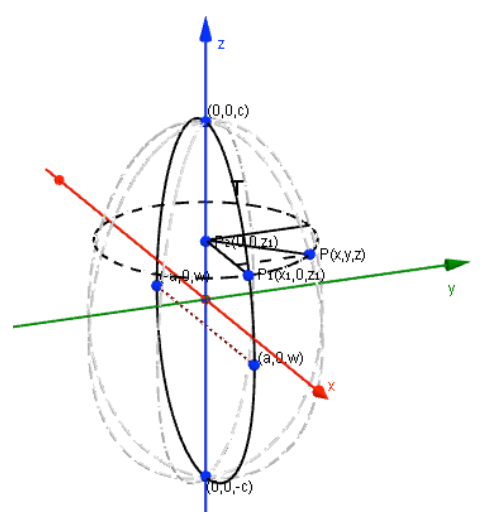

Fig. 4. The $T$ curve in $x z$-plane rotated on $z$-axis.

By using the construction steps are similar to previous session, then the equation of egg-shaped surface where the mid-point of major axis is at $O(0,0,0)$ and the z-axis is as rotation axis, is $\frac{x^{2}}{a^{2}}\left(1+\frac{2 w z+w^{2}}{c^{2}}\right)+\frac{y^{2}}{a^{2}}\left(1+\frac{2 w z+w^{2}}{c^{2}}\right)+\frac{z^{2}}{c^{2}}=1$

where $c>a>0 ; 0<|w|<c ; a, c, w$ constants.

Whereas, the equation of egg-shaped surface where the mid-point of major axis is at $Q(p, q, r)$, is

$\frac{(x-p)^{2}}{a^{2}} f(z)+\frac{(y-q)^{2}}{a^{2}} f(z)+\frac{(z-r)^{2}}{c^{2}}=1$

where $f(z)=\left(1+\frac{2 w(z-r)+w^{2}}{c^{2}}\right)$.

The volume of egg-shaped solid

The volume of the egg-shaped solid can be obtained by using Hügelschaffer's egg-shaped curve on $x y$-plane that is rotated on $x$-axis. By using the disk method of the volume integral [8], then we obtained the volume formula of the egg-shaped solid, that is

$V=\pi b^{2}\left[\left(a^{2}-\frac{\left(a^{2}+w^{2}\right)^{2}}{4 w^{2}}\right) \frac{1}{w} \ln \left(\frac{a+w}{a-w}\right)+\frac{a\left(a^{2}+w^{2}\right)}{2 w^{2}}\right]$.

The egg-shaped surface area

In calculus, to find the surface area of solid that has symmetric axis or is obtained by rotating a curve, we can use formula [8] :

$$
A=2 \pi \int_{a}^{b} f(x) \sqrt{1+\left(f^{\prime}(x)\right)^{2}} d x
$$

By stating the Eq. 1 in the form of explicit function $y=f(x)$, and finding the differensial $f^{\prime}(x)$, then substituted to Eq. (9), we obtained the integrand of the surface area of egg-shaped, that is

$A=2 \pi b \int_{-a}^{a} \frac{\sqrt{\left(a^{2}-x^{2}\right)\left(a^{2}+2 w x+w^{2}\right)^{3}+b^{2}(w+x)^{2}\left(w x+a^{2}\right)^{2}}}{\left(a^{2}+2 w x+w^{2}\right)^{2}} d x$

Because the Eq. 10 is difficult to be solved analytically, furthermore the surface area of the eggshaped (Eq. 10) is calculated numerically.

\section{Visualisation of the egg-shaped surface equations}

Visualisation of the egg-shaped surface equations in this paper use GeoGebra software. The Eq. 2 to Eq. 7 are changed in the parametric form : 


$$
\begin{aligned}
& x=a \cos t \\
& y=\frac{a b \sin t \cos u}{\sqrt{a^{2}+2 a w \cos t+w^{2}}} \\
& z=\frac{a b \sin t \sin u}{\sqrt{a^{2}+2 a w \cos t+w^{2}}} ;
\end{aligned}
$$

with $0 \leq t \leq \pi$ and $0 \leq u \leq 2 \pi$.

For examples, the results of the visualisation of the egg-shaped surface equations (Eq. 2 to Eq. 7) with $a=2,685, b=1,835$ and $w=0,45$, respectively are as follow :

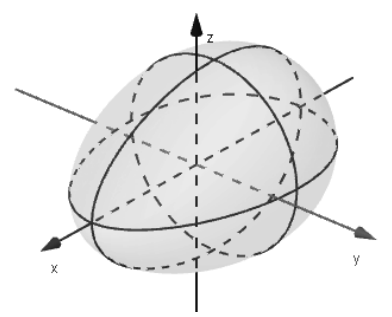

Fig. 5. Egg-shaped surface from Eq. 2.

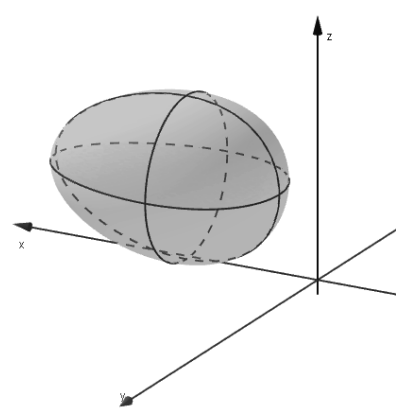

Fig. 6. Egg-shaped surface from Eq. 3 with $Q(2,3,3)$.

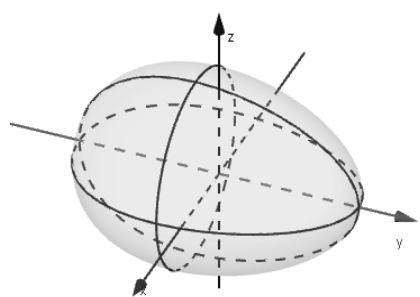

Fig. 7. Egg-shaped surface from Eq. 4.

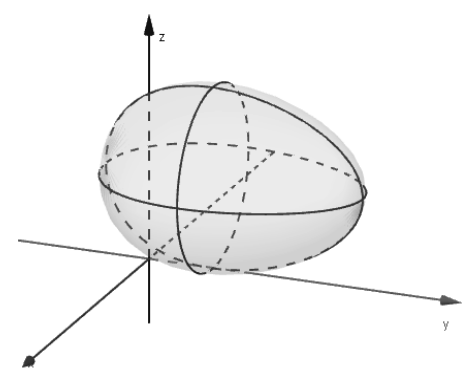

Fig. 8. Egg-shaped surface from Eq. 5 with $\mathrm{Q}(3,2,3)$.

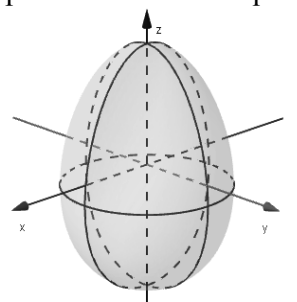

Fig. 9. Egg-shaped surface from Eq. 6.

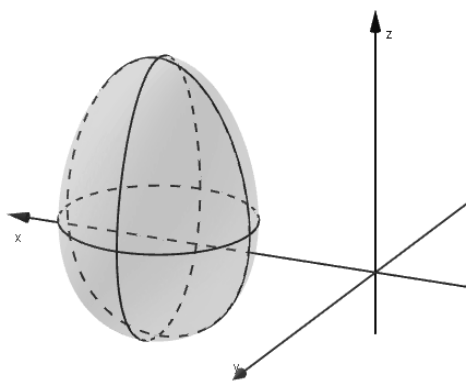

Fig. 10. Egg-shaped surface from Eq. 7 with $Q(3,3,2)$.

If the parameter values of $a, b$ and $w$ are substituted to Eq. 8 and Eq. 10, we obtained the volume is $V \approx 37,657$ and surface area is $A \approx 4,84$. The corresponding between parameter $a$ and $b$ and parameter $a$ and $w$ to the oval egg-shaped surface are $0,681 \leq \frac{b}{a} \leq 0,815$ and $0,0292 \leq \frac{w}{a} \leq 0,1675$. This interval is measured from 63 chicken eggs. The oval egg-shaped surface can be seen on the Fig. 5 to Fig. 10 where the surfaces is oval or ovoid.

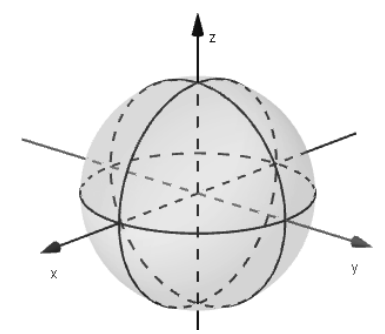

Fig. 11. Sphere with radius 2.

The effect of the parameter values to the eggshaped surface is as shown on Figure 11 to Figure 14. The Eq. 6 is sphere if we choose $a=b>0$ and $w=0$. It is as shown on Fig. 11. Whereas, the Eq. 6 is ellipsoid if we choose $a>b>0$ and $w=0$, that is shown on Fig. 12.

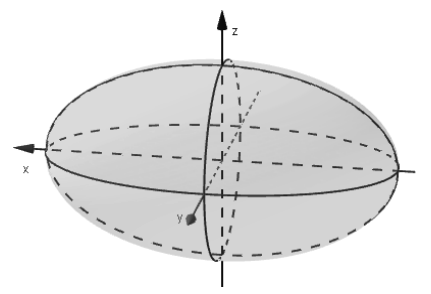

Fig. 12. Ellipsoid.

If we choose the parameter values of $a, b$ and $w$ out of the interval of oval egg-shaped, we can get the surface as shown on Fig. 13. That is, when the value of parameter $a$ is more and more large, then the surface does not represent oval egg-shaped. 


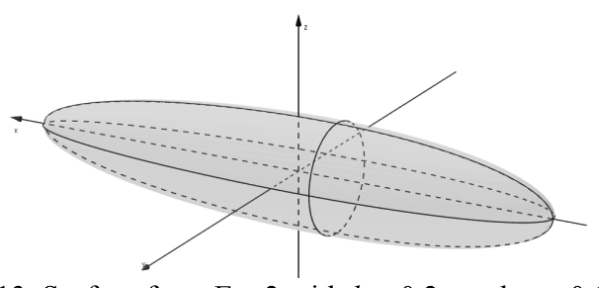

Fig. 13. Surface from Eq. 2 with $b=0,2 a$ and $w=0,1047 a$.

Meanwhile, if the condition of Eq. 6 is not sufficed, the visualisation of the surface is not eggshaped. It is as shown on Fig. 14.

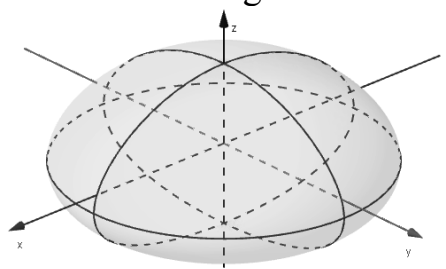

Fig. 14. Surface from Eq. 6 with $a=3, c=2$ and $w=0,44$.

\section{CONCLUSION}

Based on discussion, the effect of the parameter values of the egg-shaped surface equations toward the visualization results are if the values of parameter $a$ and $b$ are constant and $w$ variables, then the shaped surface be far from oval when the value of $w$ is more and more large. Otherwise, if the value of $w$ is more and more small, then the shaped surface close to ellipsoid. This conclusion can be satisfy if $a$ and $b$ are constant and sufficed the interval $0,681 \leq \frac{b}{a} \leq 0,815$.

If the values of $a$ and $w$ are constant and $b$ variables, then the shaped surface close to sphere when the value of $b$ close to $a$. Otherwise, when the value of $b$ is more and more small, the shaped surface close to ellipsoid if the value of $w$ close to zero, and the shaped surface close to oval if the value of $b$ is in the interval of $0,681 \leq \frac{b}{a} \leq 0,815$. This conclusion is hold if the values of $a$ and $w$ are in the interval of $0,0292 \leq \frac{w}{a} \leq 0,168$.

If the values of $b$ and $w$ are constant and $a$ variables, the shaped surface be far from oval when the value of $a$ is more and more large. Meanwhile, if the values of $a=b>0$ and $w=0$, then the shaped surface is sphere. Whereas, if the values of $a>b>0$ and $w=0$, then the shaped surface is ellipsoid.

\section{REFERENCES}

[1] Nishiyama, Y., The Mathematics of Egg Shaped, Department of Business Information, Faculty of Information Management, Osaka University of Economics, Osaka, Japan, 1986.

[2] J. Koller, Egg Curves and Ovals, 2000. [Online]. Available : www.mathematische-basteleien.de. Accessed 20 Pebruary 2015.

[3] Obradovic M. et al., Generating Curves of Higher Order Using the Generalization of Hugelschaffer's Egg Curve Construction, "Politehnica" University of Timisoara, Faculty of Civil Engineering, Timisoara, Romania, pp. 110-115, 2013.

[4] Obradovic M. et al., Conic Sections of a Type of Egg Curve Based Conoid, Proceeding MonGeometrija 2010, Faculty of Architecture in Belgrade, Serbia, pp. 447-466, 2010.

[5] Petrovic M. et al., Suitability Analysis of Hugelschaffer's Egg Curve Application in Architectural and Structure's Geometry, Buletinul Instutului Politehnic din Iasi, Universitatea Tehnica Gheorghe Asachi, Vol. III, pp. 115-122, 2011.

[6] N. Yamamoto, Equation of Egg Shaped Curves, 2007, [Online]. Available : www.geocities.jp. Accessed 20 Pebruary 2015.

[7] Petrovic M. et al., The Complement of the Hugelschaffer's Construction of the Egg Curve, Proceeding 25th National and 2nd International Scientific Conference, MonGeometrija 2010, Vlasina, Serbia, pp. 520-531, 2010.

[8] Larson, R., Calculus 9th edition, Brooks/Cole, USA, 458, 2010.

[9] Chapra, S. C., Applied Numerical Method with MATLAB for Engineers and Scientists $3^{\text {rd }}$ ed., Mc Graw Hill, New York, 510, 2012. 